\section{Alopecia in patients with vitamin D-resistant rickets type-II*}

Karisa Farias Miksza ${ }^{1}$

Gabriel Martinez Andreola ${ }^{2}$

Fabiane Mulinari Brenner ${ }^{1}$ Paula Hitomi Sakiyama ${ }^{3}$

\section{DOI: http://dx.doi.org/10.1590/abd1806-4841.20175706}

Dear Editor,

Rickets is the most common bone disease in childhood. ${ }^{1}$ The vitamin D-dependent type II (VDDR-II) is a rare and severe type of hereditary rickets described, in references worldwide, as affecting only 50 families. $^{2}$ This report is designed to describe the first hereditary rickets case reported in the city of Curitiba, in which the patient displays alopecia as a frequent clinical dermatological manifestation.A 12-year old female patient born from unrelated parents, presented, since she was three months old, alopecia and eyelash and eyebrow loss, as well as delayed motor development, deformed lower limbs, due to reduced longitudinal growth, long bones and dental alterations. At seven months of age, after being diagnosed with vitamin $\mathrm{D}$ resistant hypophosphatemic rickets, the use of vitamin D, elemental calcium, calcitriol, magnesium mill, and hydrochlorothiazide was started to control the calcium metabolism dysfunction. Another case was reported in the family, starting at the same age, involving the patient's mother's first cousin. During physical examination, the patient's short stature, deformed lower limbs, absence of hair on scalp, thin eyebrow rarefaction, and sharp triangular teeth were noticed (Figures 1 and 2). A dermoscopic evaluation of the scalp revealed the presence of irregular white dots, suggesting the absence or hypoplasia of the follicle structure, and the presence of regular pin point white dots, suggesting an acrosyringium opening, with no other follicular signs (Figure 3). Scalp biopsy showed non-scarring alopecia with without folicular Units. Alopecia areata hypothesis was ruled out after histological and dermoscopic evaluation, due to the absence of black dots, exclamation marks and inflammation. No improvement was observed despite severe treatments incluing $5 \%$ topical minoxidil and $0,05-0,1 \%$ dinitrochlorobenzene. Rickets is the most common childhood metabolic bone disease, characterized by a decreased mineralization of the growth epiphyseal plate. ${ }^{1}$ Failure in the mineralization process occurs due to inadequate extracellular concentration of calcium and phosphate ions, as well as to the involvement of the action of the

\section{Received on 17.02 .2016}

Approved by the Advisory Board and accepted for publication on 09.08.2016

* Work developed at Hospital de Clínicas - Universidade Federal do Paraná (UFPR),

Curitiba, PR, Brazil

Financial support: None.

Conflict of interests: None.

Dermatology Service at the Universidade Federal do Paraná (UFPR), Curitiba, PR Brazil.

Universidade Federal do Paraná (UFPR) „, Curitiba, PR, Brazil.

Universidade Católica do Paraná (PUCPR), Curitiba, PR, Brazil.

C2017 by Anais Brasileiros de Dermatologia elements responsible for their absorption, especially vitamin D. Alterations in this vitamin, if genetically defined, determine two rare types of rickets, both autosomal recessive: vitamin D-dependent type I (mutations on the 1-alpha hydroxylase enzyme gene -12q13) and vitamin D-dependent type II, or vitamin D-resistant (mutation on the vitamin D receptor gene -12q14). The case reported here demonstrates a vitamin D-dependent type II situation., ${ }^{2,3}$ Vitamin D-dependent rickets type II (VDDRII) results from the mutation on the vitamin D receptor gene (VDR), which is expressed in several target organs (intestines, kidneys, bones, parathyroid, and hair follicle). ${ }^{4,5}$ Immunohistochemical study demonstrated the VDR expression in keratinocytes of the outer rooth sheath hair and in dermal papillae fibroblasts, with higher immunoreactivity levels during the anagen and catagen phases. Therefore, when the VDR system is not intact, inadequate differentiation of the pilous follicle takes place, separate from electrolyte homeostasis, which would explain the occurrences of these signals, specifically in VDDR type II, and which would most likely result in the maintenance of alopecia, despite the replacement of vitamin D and calcium. However, this mechanism has not been fully elucidated. ${ }^{5}$ VDDRII's clinical spectrum varied based on the mutation type and on the receptor's residual activity level; nevertheless, it is usually asymptomatic at birth and during the first two years of life, displays delay neuromotor development , bone abnormalities, and frequent infections. Although the main clinical manifestations are similar in different types of rickets, there are specific clinical signals, and full or partial alopecia is a marker of forms associated with the defect in VDR. It represents one of the first symptoms, occurs in 2/3 of the cases, and is, in general, universal. Its occurrence is associated to poor prognosis, and has an important

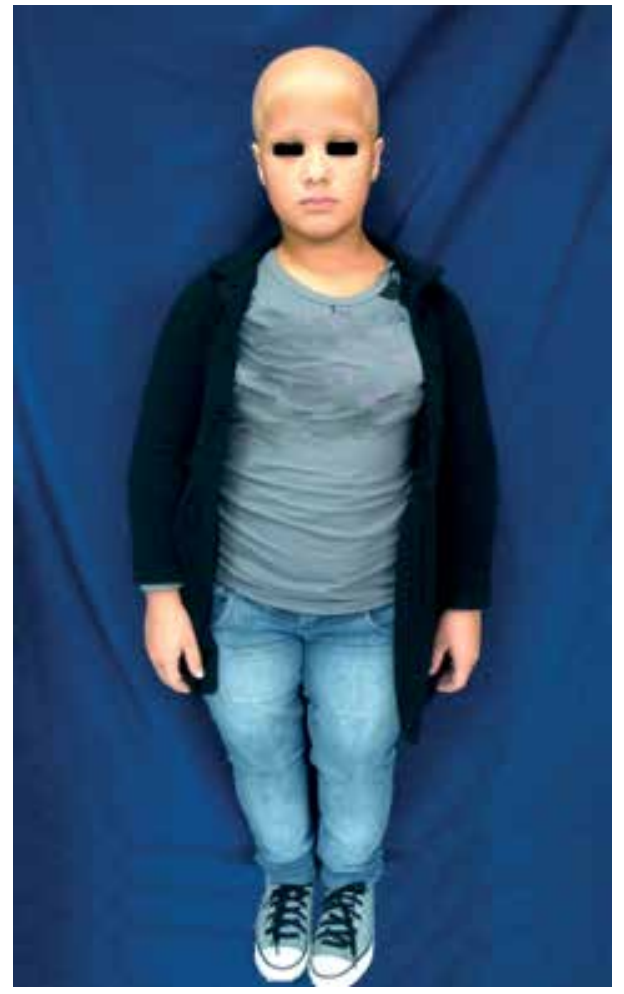

Figure 1: Low stature 


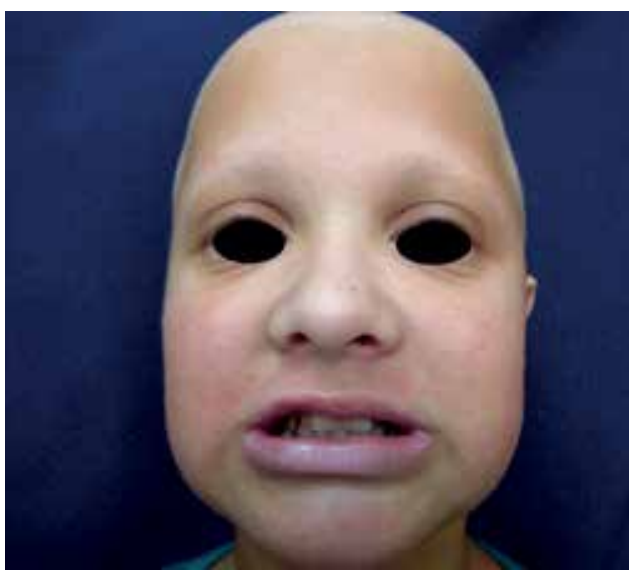

Figure 2: Clinical aspect of total alopecia and dentition

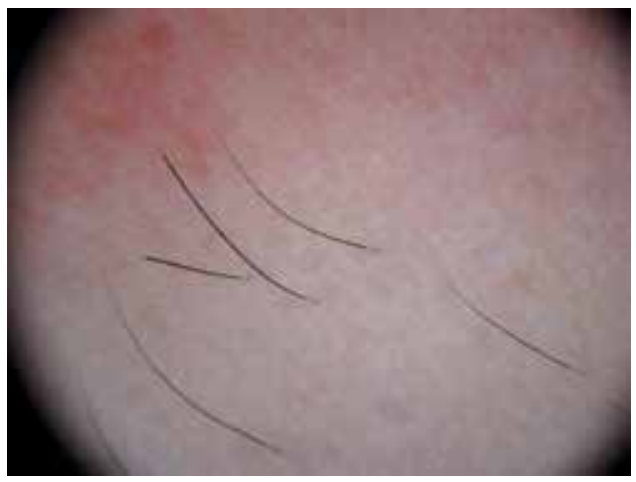

FiguRE 3: Eyebrow dermoscopy: sparse vellus hair on the face

contribution for establishing this type of disease's hereditary nature due to the significant association with VDDRII. Histological exam performed on the alopecia area demonstrates thinning and hypoplastic hair follicles, with no specific scarring or inflammatory characteristics. ${ }^{1}$ Frequent biochemical alterations contribute to the diagnosis: high calcitriol with normal calcidiol, hypocalcemia, increased alkaline phosphatase, and phosphaturia and hypophosphatemia. ${ }^{1,2,3}$ Treatment, which is based on a supplementation consisting of a high dose (30 to $60 \mathrm{ug} /$ day) of vitamin $\mathrm{D}$ and calcium taken orally (up to $3 \mathrm{~g} /$ day), is essential before reaching three years of age in order to avoid potentially fatal complications. ${ }^{1}$ In the case reported here, as well as in cases described in the literature, specific VDDRII treatment, with calcium and vitamin D supplementation, as well as several options for alopecia treatment, have not contributed to the clinical improvement of alopecia. $\square$

\section{REFERENCES}

1. Santos R, Neves S, Gomes C, Neves F, Correia AJ. Raquitismo Vitamina D Dependente Tipo II. Acta Med Port. 2009;22:8616.

2. Malloy PJ, Wang J, Srivastava T, Feldman D. Hereditary 1,25-dihydroxyvitamin D-resistant rickets with alopecia resulting from a novel missense mutation in the DNAbinding domain of the vitamin D receptor. Mol Genet Metab. 2010;99:72-9

3. Lisse TS, Saini V, Zhao H, Luderer HF, Gori F, Demay MB. The vitamin $\mathrm{D}$ receptor is required for activation of $\mathrm{cWnt}$ and hedgehog signaling in keratinocytes. Mol Endocrinol.
2014;28:1698-706

4. Casey G, McPherson T, Kini U, Ryan F, Taibjee SM, Moss C, et al. Hereditary vitamin D-resistant rickets presenting as alopecia. Pediatr Dermatol. 2014;31:519-20.

5. Mechica José B. Raquitismo e osteomalacia. Arq Bras Endocrinol Metab. 1999;43:457-66.

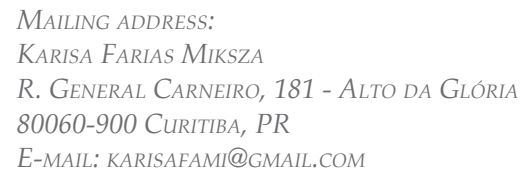

How to cite this article: Miksza KF, Brenner FM, Andreola GM, Sakiyama $P H$. Alopecia in patients with vitamin D-resistant rickets typeII. An Bras Dermatol. 2017;92(2):286-7.

\section{Exuberant angiosarcoma*}

Amanda Brilhante Pontes ${ }^{1}$ Daniel Lago Obadia ${ }^{1}$

Juliana Lacerda Reis Ucelli ${ }^{1}$ Leninha Valério do Nascimento ${ }^{1}$

Dear Editor,

We report the case of a white, female patient, 78 years of age, presenting an erythematous rash on the face for the past two months, which was asymptomatic but was gradually increasing in size. She had used antibiotics and acyclovir, with no improvement in the lesion. Dermatological examination revealed an erythematous-violaceous plate, with revealed an, and accentuated follicular ostia, affecting the scalp, the upper eyelid, and preauricular, frontal, and mandibular areas, on the left side (Figure 1a). An incisional biopsy performed revealed preserved epidermis and dermis with diffuse infiltration of vascular clefts slits, which dissect collagen fibers, corresponding to pleomorphic ectasic vascular channels with atypical endothelial cells (Figure 2). The immunohistochemical pattern revealed positive CD31, CD34, Vimentin, and Ki67, confirming angiosarcoma (Figure 3).

We chose to start chemotherapy with a weekly $80 \mathrm{mg} / \mathrm{m}^{2}$ dose of paclitaxel. After six weeks, a nearly full remission of the skin lesion was observed, leaving only residual hypochromia areas (Fig-

Received on 02.04 .2016

Approved by the Advisory Board and accepted for publication on 01.08.2016

* Work developed at the Tropical Dermatology Service at the Hospital Central do Exército (HCE), Rio de Janeiro, RJ, Brazil.

Financial support: None.

Conflict of interests: None.

Tropical Dermatology Service, Hospital Central do Exército (HCE), Rio de Janeiro, RJ, Brazil.

(C2017 by Anais Brasileiros de Dermatologia 